\title{
High Expression of PRMT6 Associated With Prognosis and Immune Infiltration in Glioma
}

\section{Yi Yang}

Department of Neurosurgery, First Affiliated Hospital of China Medical University, Shenyang

Zhenshuang Wang

Department of Orthopedics, First Affiliated Hospital of China Medical University, Shenyang

\section{Shengrong Long}

Department of Neurosurgery, First Affiliated Hospital of China Medical University, Shenyang

\section{Jinhai Huang}

Department of Neurosurgery, First Affiliated Hospital of China Medical University, Shenyang

\section{Chengran Xu}

Department of Neurosurgery, First Affiliated Hospital of China Medical University, Shenyang

\section{Mingze Yao}

Department of Neurosurgery, First Affiliated Hospital of China Medical University, Shenyang

Lun Li

Department of Neurosurgery, Anshan Hospital of the First Hospital of China Medical University, Anshan

Guangyu Li ( $\sim$ liguangyu1972@sina.com )

Department of Neurosurgery, First Affiliated Hospital of China Medical University, Shenyang

\section{Research}

Keywords: Protein arginine methyltransferase 6 , Glioma, Biomarker, The Cancer Genome Atlas(TCGA), Immune infiltration, Immunohistochemical experiment, Nomogram

Posted Date: December 23rd, 2020

DOI: https://doi.org/10.21203/rs.3.rs-133309/v1

License: (a) (1) This work is licensed under a Creative Commons Attribution 4.0 International License. Read Full License 


\section{Abstract}

Background: Gliomas are characterised by easy invasion of surrounding tissues, high mortality and poor prognosis. Moreover, with the increase of grade, the prognosis of glioma is increasingly poor and not optimistic. Therefore, biological markers for glioma are needed in clinical work, which can be utilized to detect and evaluate the situation and prognosis of glioma patients. Many studies have found that the protein arginine methyltransferase 6 (PRMT6) expression is elevated in various tumors and is associated with patient prognosis. However, the role of PRMT6 in glioma has not been reported or analyzed.

Methods: In this study, we used a variety of tumor related databases to analyze the mechanism of PRMT6 in tumors, especially gliomas, from the perspective of bioinformatics, and carried out relevant experimental verification with tumor tissues extracted from patients during surgery. In addition, we analyzed the relationship between PRMT6 expression and immune infiltration and immunerelated cells, and discussed the possible mechanisms. We also discussed the role of PRMT6 expression in glioma from the perspectives of mutation, clinical indicators, enrichment analysis, and immunohistochemical results.

Results: PRMT6 is significantly differentially expressed in a variety of tumors and is associated with survival and prognosis. Especially in gliomas, the expression of PRMT6 gradually increased with the increase of grade. In addition, PRMT6 can be used as an independent prognostic risk factor in the nomogram and has been verified in a variety of databases.

Conclusions: Our results indicate that high expression of PRMT6 is a potential biomarker for predicting glioma prognosis and progression.

\section{Introduction}

Among the central nervous system, gliomas are the most common and aggressive tumors, accounting for $70-80 \%$ of primary malignancy tumors[1-3]. Low grade glioma (LGG) is a kind of glioma with slow growth at the initial stage of development[4], while glioblastoma multiforme (GBM) is easy to invade and has a high recurrence rate[5]. Currently, treatments of gliomas are mainly surgery and chemoradiotherapy, and the relationship between surgical resection range and prognosis is controversial. However, gliomas are more likely to recrudesce and are less effective in treatment, leading to a risk of postoperative seizures[6, 7]. At the molecular level, gliomas molecular markers mainly include IDH mutation, 1p/19q co-deletion[8], MGMT promoter methylation[9] and TP53 mutation. However, the pathogenesis and molecular of gliomas are still poorly understood and much research is needed. It arises out of considerable significance and the urgent need to study tumor markers from the molecular level. In recent years, the important role of immune cell infiltration in gliomas has been paid more and more attention. Some studies have indicatesd that infiltration of immune cells in gliomas can promote the invasion and progression of gliomas[10,11]. Such as macrophages had a high proportion in tumor tissue, and the interaction of tumour-infiltrating $T$ cells and multiple signalling pathways remained to be developed, for example, the inhibitory effect of PD-1 in the inflammatory response[12]. Based on these immune mechanisms, immunotherapy for gliomas remains to be further explored.

In the nucleus, protein arginine methylation is a post-translational modification and is associated with signal transduction[13]. The protein arginine methyltransferase(PRMT) catalyzes this process. There are eleven known types of PRMT, which fall into three categories[14]. PRMT6 produces asymmetric dimethylarginine and has unique characteristics of self-methylation[15]. It is responsible for the methylation of histone H3R2 and widely expresses in various tissues of the body[16]. As a result, PRMT6 had been studied in many tumors. According to PRMT6's unique biological role, many studies had concluded that it promoted the development of cancer through different signalling pathways or inflammatory cells[17]. Recent studies had reported that PRMT6 promoted cell proliferation of endometrial cancer through the AKT/mTOR pathway and further promoted carcinogenesis[18]. Additionally, there were related studies in lung cancer, hepatocellular carcinoma, colon cancer and prostate cancer[19]. However, the mechanism of PRMT6 in gliomas is not precise, and immune-related studies are insufficient.

At present, there is the absence of research on the role of PRMT6 in gliomas. Based on multiple tumor-related public databases, we explored the potential mechanism and role of PRMT6 in gliomas from the perspectives of immune mechanism, epigenetics and clinical prognosis. Our analysis revealed that PRMT6 is a potential tumor marker for glioma and provides important evidence for epigenetic and immunological correlation of it.

\section{Materials And Methods}


Genotype-Tissue Expression (GTEx) database mainly includes the expression of genes in healthy human tissues and organs [20]. We obtained the expression of PRMT6 in a variety of normal tissues from this database. In Figure 1a, the X-axis lists 31 kinds of tissues and the Y-axis represents the expression amount of PRMT6 calculated by Log2(TPM+1).

The Cancer Genome Atlas(TCGA) database is a comprehensive database, containing not only the molecular level data of dozens of primary tumors, but also the pertinent clinical data. First, in the TCGA database, we selected the data set with the number of normal samples greater than equal 5. We performed a differential analysis among the 18 tumors (Figure 1b). Next, we combined the normal samples from the GTEx database with those from the TCGA database, and made further difference analysis of PRMT6. Taking 24 tumor samples as X-axis, the expression of PRMT6 was calculated by Log2(TPM+1) as Y-axis (Figure 1c).

Cancer Cell Line Encyclopedia (CCLE) database, which mainly records the genetic characteristics of cell lines, sequence more than 900 cancer cells from more than 30 human tissues. In this study, data from multiple tumor cell lines were downloaded from the CCLE database, and PRMT6 expression levels in 21 tissues were compared(Additional file 1: Figure S1). Taking 1019 samples and 21 tissues as X-axis, the expression of PRMT6 was calculated by Log2(TPM+1) as Y-axis.

\section{Analysis of gene expression and survival prognosis}

In our study, gene expression profile from TCGA database were utilized to analyze the relationship between PRMT6 expression in 33 tumours and clinical prognostic indicators, including overall survival(OS), disease-specific survival(DSS) and progression-free survival(PFS). We used Kaplan-Meier survival analysis curve and forest map respectively to visualize the relationship between them (Figure 2).

\section{Analysis of gene expression and immune estimation}

CIBERSORT is a software to analyze immune cell infiltration[21]. We downloaded marker for gene expression of 22 immune cells from the website. In this study, scores of immune infiltration were collected and the correlation between the expression of PRMT6 in these tumors and the immune cells infiltration (Additional file 2: Figure S2). Taking immune cells infiltration score of CIBERSORT as the Xaxis, the expression of PRMT6 was calculated by Log2(TPM+1) as Y-axis (Figure 3a).

We used the Estimate package in R software to conduct a comprehensive analysis on the scores of the total contents of immune cells and stomatal cells in each type of tumor (Additional file 3: Figure S3). Taking the corresponding scores as X-axis, the expression of PRMT6 was calculated by Log2(TPM+1) as Y-axis, and the distribution of each score was described by a density curve (Figure 3c-f).

We obtained the information of more than 40 immune checkpoint genes and studied the relationship between the expression of PRMT6 and the expression of immune checkpoint genes(Figure $3 b$ ).

TISIDB database is an open online tool that integrates a variety of tumor immunology resources[22]. We used this database to analysis the relationship between PRMT6 expression in a variety of tumors and immune or molecular subtypes (Additional file 4: Figure S4).

\section{Analysis of gene expression and mutation}

The number of gene mutations in each tumor was obtained from the TCGA database and corrected by comparing the total length of exons. We tested the correlation between PRMT6 expression and tumor mutational burden (TMB) and visualized the relationship between them through radar map (Figure 4a).

Microsatellite instability (MSI) is a change in the length of a microsatellite due to insertion or deletion of duplicate units in the tumor compared to normal tissue. We visualized the correlation between the expression of PRMT6 in tumors and MSI by radar map (Figure $4 b)$.

\section{Analysis of gene expression and clinical indicators}

Chinese Glioma Genome Atlas (CGGA) is a complete genome sequencing database for glioma patients in China. We obtained the clinical information and PRMT6 gene expression information of 325 glioma patients from the CGGA database[23, 24]. Then we 
analyzed the clinical information of CGGA and TCGA databases respectively, and analyzed the expression of PRMT6 and its correlation (Figure 5a-j). Kruskal-Wallis test and Mann-Whitney test were used to determine whether gene expression was different among various clinical indicators. The $\mathrm{X}$-axis is the classification of various clinical indicators and the $\mathrm{Y}$-axis is the gene expression level.

\section{Construct and verify the nomogram}

We used the high and low expression of PRMT6 as an independent prognostic risk factor and combined it with other common risk factors to construct a nomogram (Figure 6c). Risk factors analyzed in this study included age, gender, tumor grade, IDH mutation status, 1p19q co-deletion status, chemotherapy status, and PRMT6 expression level. To verify whether PRMT6 can act as an independent prognostic risk factor, we first plotted the survival analysis curve using Kaplan-Meier method (Figure 6a-b). Then univariate Cox and least absolute shrinkage and selection operator (LASSO) regression model were used to screen all variables. TCGA database was used as the training set and CGGA database as the verification set, and consistency index (C-index) and calibration curve were used to evaluate the nomogram (Figure 6d-e).

\section{Gene Set Enrichment Analysis(GSEA)}

GSEA is a computational method used to determine whether a predefined data set has significant consistent differences between two biological states[25]. And we downloaded GSEA software from the website. The expression dataset was the expression of PRMT6, the experimental group and the control group were set to the high expression and low expression of PRMT6, and the number of permutations was set to 1000. In the obtained results, NOM p-val and FDR q-VAL value less than 0.05 were considered to have a significant difference.

\section{Immunohistochemical analysis}

The specimens used in this study were from 32 glioma tissues and 4 glioma paracancerous tissues surgically removed from the First Affiliated Hospital of China Medical University between 2012 and 2014. The clinical information of all patients was obtained, and the survival time and survival status of patients were obtained through telephone follow-up. Of the 32 glioma cases, 20 were male and 12 were female; they ranged in age from 29 to 80, with a median age of 51; according to the grading standards of the World Health Organization (WHO), there were 12 cases of WHO II, 7 cases of WHO III and 13 cases of WHO IV; 21 deaths, with a mortality rate of $65.6 \%$; the mean follow-up time was 43.65 months.

PRMT6 antibody used in this study is rabbit anti-human polyclonal antibody from Novus, USA (No. NB 110-40713). SP hypersensitive immunohistochemical kit was used for the secondary antibody, which came from Fuzhou Maixin Biotechnology Development Limited Liability Company, China.

The experimental procedure was carried out in strict accordance with the instructions of the immunohistochemical kit. Firstly, the paraffin sections were dehydrated, and then the antigens were repaired by microwave heating. The primary antibody (dilution concentration of PRMT6 was 1:500) was added for incubation for 1 hour, and then the secondary antibody was dropped for incubation for DAB color development. The blank control group was set to replace primary antibody with phosphate buffer saline. Through the identification of pathologists in our hospital, the staining intensity and positive cell rate of each section were graded by semi-quantitative integration method, and then the product of the two scores was used to obtain the positive intensity (Table 1). Immunohistochemical scores: 0-1 is negative expression, 2-4 is low expression, and 6-9 is high expression.

\section{Table 1: Grading criteria}

\begin{tabular}{|lll|}
\hline Immunohistochemical score & Staining intensity of positive cells & Percentage of positive cells \\
\hline 0 & No staining & $<5 \%$ \\
\hline 1 & Weak staining & $5 \%-25 \%$ \\
\hline 2 & Medium staining & $26 \%-75 \%$ \\
\hline 3 & Strong staining & $>75 \%$ \\
\hline
\end{tabular}


We used the Log-rank test and Cox analysis to calculate OS, DSS and PFS. Spearman method, Wilcox test and Kruskal test were used to determine the correlation coefficient between the expression of PRMT6 and other indicators, and calculate the corresponding Pvalue, hazard ration(HR) and $95 \%$ confidence interval $(95 \% \mathrm{Cl})$. All $\mathrm{P}$ values less than 0.05 were examined statistically significant. In this study, R-3.6.3 was used for data analysis and visualization, and Adobe illustrator CC 2019 was used for figures processing. Immunohistochemical data processing and graphics rendering was done using GraphPad Prism 8.

\section{Results}

\section{The expression of PRMT6 in different human tissues and cancers}

To clarify the differences between PRMT6 in healthy tissues and tumor tissues, we performed a visual analysis of the expression of PRMT6 in healthy tissues. As can be observed in Figure 1a, PRMT6 expression level was low in healthy brain tissues. Then we also analyzed the expression of PRMT6 in different tumors and normal tissues. Tumors with significant positive associations included: Adrenocortical carcinoma (ACC), Bladder Urothelial Carcinoma (BLCA), Breast invasive carcinoma (BRCA), Cervical squamous cell carcinoma and endocervical adenocarcinoma (CESC), Colon adenocarcinoma (COAD), Esophageal carcinoma『ESCA囚, Glioblastoma multiforme (GBM), LGG, Liver hepatocellular carcinoma (LIHC), Lung adenocarcinoma(LUAD), Lung squamous cell carcinoma (LUSC), Ovarian serous cystadenocarcinoma (OV), Pancreatic adenocarcinoma (PAAD), Prostate adenocarcinoma (PRAD), Skin Cutaneous Melanoma (SKCM), Stomach adenocarcinoma (STAD), Testicular Germ Cell Tumors (TGCT), Thyroid carcinoma (THCA), Uterine corpus endometrial carcinoma (UCEC), Uterine Carcinosarcoma (UCS). There were also some negatively correlated tumors: Kidney renal clear cell carcinoma (KIRC), Acute Myeloid Leukemia (LAML), Kidney Chromophobe $\mathbb{K} \mathrm{KICH \bigotimes ,} \mathrm{Kidney} \mathrm{renal} \mathrm{papillary} \mathrm{cell} \mathrm{carcinoma}$ (KIRP).

\section{Analysis of survival prognosis}

We used clinical information from the TCGA database to study the correlation between PRMT6 and survival prognosis. It can be seen from the forest graph of OS, DSS and PFI that the expression of PRMT6 is a risk factor in BLCA, LGG and UCEC and a protection factor in BRCA. In addition, PRMT6 expression was divided into high and low groups, and survival analysis curves were drawn. In OS analysis, the high and low expression of PRMT6 was significant in LGG and UCEC. In DSS analysis, the high and low expression of PRMT6 was significant in LGG, LUAD and UCEC. In PFI analysis, the high and low expression of PRMT6 was significant in LGG, COAD and LUAD. These evidence suggest that PRMT6 has important reference value for prognosis in tumors.

\section{Multidimensional immune correlation analysis}

To study the effect of PRMT6 on immunity level, we analyzed its interaction with different immune infiltrating cells, immune microenvironment, immune subtype and immune checkpoint genes from different angles. As we can see from Additional file 1 Figure S1, PRMT6 expression in BRCA, CESC, Head and Neck squamous cell carcinoma(HNSC), KIRC, LGG, LIHC, PAAD, SKCM, Thymoma(THYM), UCEC significantly correlation with the immune cells existed. These results indicate that PRMT6 expression is strongly correlated with immune cells in tumors.

Next, we analyzed the relationship between PRMT6 expression and stromal cell and immune cell score in tumors. The stronger the correlation, and the higher the score, the more significant the proportion of stromal cells and immune cells in the tumor tissue. We can see from the Additional file 2 Figure S2, in BLCA, CESC, GBM, LAML, LUSC, OV, Pheochromocytoma and Paraganglioma(PCPG), PRAD, Sarcomav(SARC), STAD, TGCT, THCA, THYM and UCEC, gene expression and immune cells or stromal cells of score was negatively correlated. And the interesting thing is that it's only positive in LGG, which can further prove the intrinsic relationship between PRMT6 expression in $\mathrm{g}$ and immune cells.

Also, we analyzed the expression relationship between PRMT6 and 47 common immune checkpoint genes. From Figure 3b, we can see that the expression of PRMT6 in a variety of tumours have a strong correlation with immune checkpoint genes.

Finally, we used the TISIDB online tool to analyze the relationship between gene expression and immune or molecular subtypes(Additional file 3 Figure S3). The results show that the expression of LGG is significantly higher than others.

\section{Mutation correlation analysis}


We calculated TMB in each cancer tumor, we can see from the radar map in ACC, THCA, PRAD, PAAD, OV, LGG, COAD and CESC has significantly correlation. In the MSI radar map, SKCM, PAAD, LUAD, KIRC, Lymphoid Neoplasm Diffuse Large B-cell Lymphoma(DLBC) and COAD have significant differences. In the MSI, COAD, DLBC KIRC, LUAD, PAAD, SKCM have statistically significant differences.

\section{Various clinical indicators correlation analysis}

To further elucidate the potential clinical value of PRMT6 in gliomas, we analyzed a variety of clinically common indicators including age, tumor grade, IDH mutation status, 1p19q co-deletion status, chemotherapy status. As can be seen from Figure 5, the expression level of PRMT6 in TCGA and CGGA databases was significantly correlated with a variety of clinically common risk factors.

\section{Independent prognostic risk factor analysis}

We considered high and low expression of PRMT6 as an independent risk factor. First, survival analysis curves were performed based on TCGA and CGGA data, and the results showed that the high and low expression of PRMT6 significantly affected the prognosis of the patients. Next, we constructed the nomogram by screening for a variety of independent risk factors. TCGA database was used as the training set and CGGA database as the validation set for external validation. From the results of the nomogram, PMRT6 is potentially valuable as an independent prognostic risk factor. In order to test the accuracy of the nomogram model, we calculated the C-index. The C-index of the nomogram of TCGA was 0.84 (95\% Cl:0.865-0.815), and the C-index of the nomogram of CGGA was 0.772 (95\% Cl:0.801-0.743). The calibration curves of the 1-year and 3-year survival rates of the two models were drawn respectively, and the results showed that the two models had better validation performance.

\section{Gene enrichment results}

According to the results of KEGG and GO enrichment analysis, the role of PRMT6 in gliomas is mainly related to the regulation of cell cycle, the involvement of DNA damage and repair, and the conduction of some signaling pathways. In Table 2, some representative pathways and related functions of enrichment are selected for demonstration.

\section{Table 2: Gene enrichment results}




\begin{tabular}{|c|c|c|}
\hline Gene set names & $\begin{array}{l}\text { NOM } \\
\text { p-val }\end{array}$ & $\begin{array}{l}\text { FDR } \\
\text { q-val }\end{array}$ \\
\hline \multicolumn{3}{|l|}{ KEGG gene set } \\
\hline KEGG_BLADDER_CANCER & 0 & 0.038 \\
\hline KEGG_SMALL_CELL_LUNG_CANCER & 0.002 & 0.038 \\
\hline KEGG_PANCREATIC_CANCER & 0.006 & 0.044 \\
\hline KEGG_SYSTEMIC_LUPUS_ERYTHEMATOSUS & 0.002 & 0.046 \\
\hline KEGG_P53_SIGNALING_PATHWAY & 0 & 0.031 \\
\hline KEGG_MISMATCH_REPAIR & 0 & 0.045 \\
\hline KEGG_NUCLEOTIDE_EXCISION_REPAIR & 0.006 & 0.043 \\
\hline KEGG_ECM_RECEPTOR_INTERACTION & 0.008 & 0.047 \\
\hline KEGG_PYRIMIDINE_METABOLISM & 0.002 & 0.034 \\
\hline KEGG_GLUTATHIONE_METABOLISM & 0.002 & 0.049 \\
\hline KEGG_AMINO_SUGAR_AND_NUCLEOTIDE_SUGAR_METABOLISM & 0 & 0.045 \\
\hline \multicolumn{3}{|l|}{ GO gene set } \\
\hline \multicolumn{3}{|l|}{ Cell cycle regulation } \\
\hline GO_CELL_CYCLE_G1_S_PHASE_TRANSITION & 0 & 0.029 \\
\hline GO_POSITIVE_REGULATION_OF_CELL_CYCLE_PHASE_TRANSITION & 0 & 0.033 \\
\hline GO_REGULATION_OF_CELL_CYCLE_PHASE_TRANSITION & 0.002 & 0.032 \\
\hline GO_REGULATION_OF_DNA_TEMPLATED_TRANSCRIPTION_IN_RESPONSE_TO_STRESS & 0 & 0.032 \\
\hline GO_REGULATION_OF_POSTTRANSCRIPTIONAL_GENE_SILENCING & 0 & 0.037 \\
\hline GO_REGULATION_OF_TRANSCRIPTION_FROM_RNA_POLYMERASE_II_PROMOTER_IN_RESPONSE_TO_HYPOXIA & 0.002 & 0.038 \\
\hline GO_SIGNAL_TRANSDUCTION_INVOLVED_IN_CELL_CYCLE_CHECKPOINT & 0 & 0.041 \\
\hline GO_MITOTIC_CELL_CYCLE_CHECKPOINT & 0.002 & 0.035 \\
\hline \multicolumn{3}{|l|}{ DNA damage and repair } \\
\hline GO_DNA_DAMAGE_RESPONSE_DETECTION_OF_DNA_DAMAGE & 0 & 0.042 \\
\hline \multirow[t]{2}{*}{ GO_DNA_DAMAGE_RESPONSE_SIGNAL_TRANSDUC GO_DNA_SYNTHESIS_INVOLVED_IN_DNA_REPAIR } & 0 & 0.042 \\
\hline & 0.002 & 0.041 \\
\hline GO_G1_DNA_DAMAGE_CHECKPOINT & 0.004 & 0.047 \\
\hline GO_NUCLEOTIDE_EXCISION_REPAIR_DNA_GAP_FILLING & 0.004 & 0.045 \\
\hline GO_SIGNAL_TRANSDUCTION_IN_RESPONSE_TO_DNA_DAMAGE & 0 & 0.04 \\
\hline \multicolumn{3}{|l|}{ Signal transduction } \\
\hline \multicolumn{3}{|l|}{ GO_REGULATION_OF_SIGNAL_TRANSDUCTION_BY_P53_CLASS_MEDIATOR } \\
\hline \multirow[t]{2}{*}{ GO_SIGNAL_TRANSDUCTION_BY_P53_CLASS_MEDIATOR } & 0.004 & 0.042 \\
\hline & 0.002 & 0.039 \\
\hline GO_TUMOR_NECROSIS_FACTOR_MEDIATED_SIGNALING_PATHWAY & 0 & 0.042 \\
\hline
\end{tabular}


Gene sets with NOM p-val and FDR q-value<0.05 are considered as significant.

\section{Immunohistochemical results}

Immunohistochemical results showed that PRMT6 protein was positively expressed in the nucleus and was brownish yellow or brown in color. The positive rate was $87.5 \%$ in glioma and $25 \%$ in normal tissue.And there was a statistical difference, with a $P$ value of 0.0055. In addition, the expression of PRMT6 in normal tissues, LGG and GBM was significantly different, and showed a significant upward trend. Based on the obtained clinical information, we drew a survival analysis curve, which further verified the significant correlation between high and low expression of PRMT6 and prognosis.

\section{Table 3: Expression of PRMT6 in glioma and normal tissues}

\begin{tabular}{|c|c|c|c|c|c|}
\hline \multirow[t]{3}{*}{ Tissue types } & \multirow[t]{3}{*}{ Number of cases } & \multicolumn{3}{|l|}{ PRMT6 } & \multirow[t]{3}{*}{$P$ value } \\
\hline & & Negative & Low & High & \\
\hline & & Expression & Expression & Expression & \\
\hline Glioma tissue & 32 & 4 & 16 & 11 & 0.0055 \\
\hline Normal tissue & 4 & 3 & 1 & 0 & \\
\hline
\end{tabular}

\section{Discussion}

PRMT6, a key epigenetic enzyme in the PRMT's family[26], is a histone modification associated with transcriptional activation and has unique self-methylation activity[27, 28]. PRMT6 participates in a variety of regulatory processes, including signal transduction and transcriptional activation[29]. At present, more and more studies have found that PRMT6 plays an important role in many tumors, but there is still a lack of relevant research in gliomas. Therefore, taken into account these conditions, we explored the expression of PRMT6 in pan-cancer and analyzed the correlation of PRMT6 expression from multiple perspectives such as immunity and mutation. At the same time, we found the close relationship and potential value of between PRMT6 and gliomas. Moreover, we validated the potential clinical value of PRMT6 from the perspective of clinical indicators. Therefore, this study takes the lead in discuss the potential value of PRMT6 as a tumor marker of gliomas and its role in immunity.

The results of our study suggest that the expression level of PRMT6 plays an important role in a variety of tumors, and this is consistent with some known studies, for example, PRMT6 could be used as a target for colon cancer in the intestinal tract[30]; the expression of PRMT6 was up-regulated in endometrial cancer samples and promoted the growth and metastasis of tumor cells by activating related pathways. Also, it affected aerobic glycolysis through signalling pathways in hepatocellular carcinoma[31]. In gliomas, the expression of PRMT6 also shows special value. From the gene expression analysis diagram, we can see that PRMT6 is low-expressed in normal brain tissue, while its expression in glioma is significantly increased. Both gene expression profile analysis and immunohistochemical results were verified.

At present, people pay more and more attention to the role of immune cells in tumor, and the type of immune cell infiltration and the subtype of immune cells are closely related to the occurrence and development of tumor[32,33]. This study found that the expression of PRMT6 in various tumor was mainly related to T cell and macrophage infiltration. In gliomas, the primary immunoinfiltrating cell are T cells CD4 memory resting and dendritic cell. More and more attention has been paid to the immunotherapy for tumor, and it is an emerging direction for the immunotherapy that stimulates the immune system to enhance the anticancer ability[34]. A number of studies have shown that immunotherapy based on autologous dendritic cells can improve patient survival[35]. Based on our results, it can be speculated that there is an intrinsic relationship between PRMT6 expression and immunotherapy targeting dendritic cells. Recent studies have shown that regulatory T cells (Treg) function in immune regulation is associated with PRMT. And there was an autoimmune response in mice with PRMT5 deficiency when Treg cells were infiltrated[36]. The results of this study showed that in gliomas, the up-regulated expression of PRMT6 was positively correlated with the infiltration of CD4 T cells, suggesting that PRMT6 is a valuable therapeutic target in immunotherapy for gliomas.

At the gene level, we also compared the correlation between the expression level of PRMT6 and the expression of some immune checkpoint genes in different tumors. In the immune checkpoint genes analogous to LGG, the genes significantly connected with the 
activation of $T$ and $B$ lymphocytes and macrophages, such as CD40, CD44, CD80 and CD86. In addition, NRP-1 gene was associated with immune regulation and cell migration and interacted with Tregs[37]. The HAVCR2 gene encoded TH1-specific cell surface proteins and was associated with regulating macrophage activation[38]. The evidence was further expressed that PRMT6 is strongly associated with infiltrating immune cells in LGG. In addition, this study linked arginine methylation with immunity and built a bridge between epigenetics and immunology.

TMB is a promising biomarker, and studies have shown that high TMB is positively correlated with immunotherapy[39]. In addition, TMB is closely related to the overall survival rate and tumor proliferative activity of glioma patients[40]. Therefore, it is an important reference factor in the treatment and prognosis of glioma. This association may be closely related to PRMT's involvement in cell cycle regulation and DNA damage, such as catalyzed cyclin expression, leading to spontaneous DNA damage, checkpoint deletion, and chromosome instability. These factors will lead to gene coding errors, base substitutions, and gene insertion or deletion errors in somatic cells, further increasing TMB and thereby promoting glioma proliferation.

In the GSEA results, we found that PRMT6 expression is related to the regulation of cell cycle, DNA damage and repair, and signal transduction in gliomas. PRMT6 plays an important role in DNA repair and regulation of DNA polymerase $\beta$ (Pol- $\beta$ ), an enzyme involved in basic repair[41]. PRMT6 is co-activated in staining as a nuclear factor to facilitate the transcription process[42]. From this evidence, we can infer that PRMT6 promotes the transcription of tumor cells in gliomas and thus increases the proliferation of tumor cells.

To verify the clinical value of PRMT6, we analyzed its association with clinically common risk factors and studied it as an independent prognostic risk factor. One of the most noteworthy was the correlation with chemotherapy. Studies have shown that PRMT6 can lead to dysfunctions of P21 ${ }^{\mathrm{CDKN} 1 \mathrm{~A}}$ (P21) in human cancers, causing it to be methylated and promoting phosphorylation of threonine 145 on P21, making cancer cells more resistant to anti-tumor drugs[43, 44]. At present, the imbalance of arginine or lysine methyltransferase in cancer has been gradually noticed[45], and new anticancer drugs targeting these sites are in the stage of clinical trials[46]. The development prospects of PRMT6 inhibitor chemotherapeutic drugs in the treatment of glioma are considerable.

\section{Conclusions}

Based on the above evidence, we can conclude that high expression of PRMT6 is a potential tumor marker for gliomas and has important predictive value for the prognosis of patients. In addition, PRMT6 may participate in the regulation of glioma cell cycle through signal transduction, promote the RNA transcription process, and further promote the proliferation and invasion of glioma cells. In clinical treatment, immunotherapy and chemotherapy are both areas to be developed.

\section{Abbreviations}

LGG: low-grade glioma; PRMT6: protein arginine methyltransferase 6; PRMT: protein arginine methyltransferase; GTEx: GenotypeTissue Expression; CCLE: Cancer Cell Line Encyclopedia; TCGA: The Cancer Genome Atlas; OS: overall survival; DSS: disease-specific survival; PFS: progression-free survival; TMB: tumor mutational burden; MSI: microsatellite instability; MMR: mis-match repair; HR: hazard ration; 95\% Cl: 95\% confidence interval; NES: normalized enrichment score; FDR: false discovery rate; BLCA: Bladder Urothelial Carcinoma; BRCA: Breast invasive carcinoma; CHOL: Cholangiocarcinoma; ESCA: Esophageal carcinoma; GBM: Glioblastoma multiforme; LUAD: Lung adenocarcinoma; LUSC: Lung squamous cell carcinoma; PRAD: Prostate adenocarcinoma; STAD: Stomach adenocarcinoma; KICH: Kidney Chromophobe; KIRC: Kidney renal clear cell carcinoma; KIRP: Kidney renal papillary cell carcinoma; THCA: Thyroid carcinoma; UCEC: Uterine corpus endometrial carcinoma; CESC: Cervical squamous cell carcinoma and endocervical adenocarcinoma; HNSC: Head and Neck squamous cell carcinoma; LIHC: Liver hepatocellular carcinoma; PAAD: Pancreatic adenocarcinoma; SKCM: Skin Cutaneous Melanoma; THYM: Thymoma; LAML: Acute Myeloid Leukemia; OV: Ovarian serous cystadenocarcinoma; PCPG: Pheochromocytoma and Paraganglioma; SARC: Sarcomav; TGCT: Testicular Germ Cell Tumors; ACC: Adrenocortical carcinoma; COAD: Colon adenocarcinoma; DLBC: Lymphoid Neoplasm Diffuse Large B-cell Lymphoma; P21:P21 CDKN1A

\section{Declarations}

\section{Acknowledgement}


All authors would like to thank the Immunization Laboratory of China Medical University for providing us with an experimental platform.

\section{Authors' contributions}

Guangyu Li selected the research topic and conducted the guidance of the process of the topic. Yi Yang wrote the article, conducted the immunohistochemical experiment and analyzed the experimental results. Zhenshuang Wang, Jinhai Huang, Chengran Xu and Shengrong Long performed data processing and image analysis. Lun Li and Minze Yao collected survival information through telephone follow-up.

\section{Funding}

The project is supported by the Science and Technology Project of Shenyang (18-014-4-03), the Science and Technology Project of the Education Department of Liaoning Province (LFWK201705) and Liaoning BaiQianWan Talents Program.

\section{Availability of data and materials}

The data used in this study are all from public databases, including GTEx database ( http://commonfund.nih.gov/GTEx/ ) , CCLE database ( http://portals.broadinstitute.org/ccle/ ), TCGA database ( https://portal.gdc.cancer.gov/ ), CIBERSORT database (https://cibersortx.stanford.edu/ ), TISIDB database (Http://cis.hku.hk/TISIDB ) , CGGA database ( http://www.cgga.org.cn/ ).

\section{Ethical approval and consent to participate}

Our study was approved by the Ethics Committee of the First Affiliated Hospital of China Medical University. All participants offered written informed consent before surgery. The study conforms to the provisions of the Declaration of Helsinki.

\section{Consent for publication}

Not applicable.

\section{Competing interests}

The authors have declared that no competing interest exists.

\section{Author details}

${ }^{1}$ Department of Neurosurgery, First Affiliated Hospital of China Medical University, Shenyang, China

${ }^{2}$ Department of Orthopedics, First Affiliated Hospital of China Medical University, Shenyang, China

${ }^{3}$ Department of Neurosurgery, Anshan Hospital of the First Hospital of China Medical University, Anshan, China

*Corresponding author. Professor Guangyu Li. Email: liguangyu1972@sina.com

\#These authors contributed equally to this study

\section{References}

1. Gallorini M, Maccallini C, Ammazzalorso A, Amoia P, De Filippis B, Fantacuzzi M, Giampietro L, Cataldi A, Amoroso R. The Selective Acetamidine-Based iNOS Inhibitor CM544 Reduces Glioma Cell Proliferation by Enhancing PARP-1 Cleavage In Vitro. International journal of molecular sciences 2019, 20(3).

2. Zhi T, Jiang K, Xu X, Yu T, Wu W, Nie E, Zhou X, Jin X, Zhang J, Wang Y, et al. MicroRNA-520d-5p inhibits human glioma cell proliferation and induces cell cycle arrest by directly targeting PTTG1. Am J Transl Res. 2017;9(11):4872-87.

3. Fan X, Wang Y, Zhang C, Liu L, Yang S, Wang Y, Liu X, Qian Z, Fang S, Qiao H, et al: ADAM9 Expression Is Associate with Glioma Tumor Grade and Histological Type, and Acts as a Prognostic Factor in Lower-Grade Gliomas. International journal of molecular sciences 2016, 17(9). 
4. Louis DN, Ohgaki H, Wiestler OD, Cavenee WK, Burger PC, Jouvet A, Scheithauer BW, Kleihues P. The 2007 WHO classification of tumours of the central nervous system. Acta Neuropathol. 2007;114(2):97-109.

5. Ryu J, Yoon N, Lee Y, Jeong J, Kang S, Seong H, Choi J, Park N, Kim N, Cho W, et al. Tristetraprolin inhibits the growth of human glioma cells through downregulation of urokinase plasminogen activator/urokinase plasminogen activator receptor mRNAs. Mol Cells. 2015;38(2):156-62.

6. Yao PS, Zheng SF, Wang F, Kang DZ, Lin YX. Surgery guided with intraoperative electrocorticography in patients with low-grade glioma and refractory seizures. J Neurosurg. 2018;128(3):840-5.

7. Han X, Xue X, Zhou H, Zhang G. A molecular view of the radioresistance of gliomas. Oncotarget. 2017;8(59):100931-41.

8. Deluche E, Bessette B, Durand S, Caire F, Rigau V, Robert S, Chaunavel A, Forestier L, Labrousse F, Jauberteau MO, et al: CHI3L1, NTRK2, 1p/19q and IDH Status Predicts Prognosis in Glioma. Cancers (Basel) 2019, 11(4).

9. Takahashi Y, Nakamura H, Makino K, Hide T, Muta D, Kamada H, Kuratsu J. Prognostic value of isocitrate dehydrogenase 1, O6methylguanine-DNA methyltransferase promoter methylation, and 1p19q co-deletion in Japanese malignant glioma patients. World J Surg Oncol. 2013;11:284.

10. Lai S, Liu Y, Lu D, Tsai C: Melatonin Modulates the Microenvironment of Glioblastoma Multiforme by Targeting Sirtuin 1. Nutrients 2019, 11(6).

11. Qian B, Pollard J. Macrophage diversity enhances tumor progression and metastasis. Cell. 2010;141(1):39-51.

12. Qian J, Wang C, Wang B, Yang J, Wang Y, Luo F, Xu J, Zhao C, Liu R, Chu Y. The IFN-gamma/PD-L1 axis between T cells and tumor microenvironment: hints for glioma anti-PD-1/PD-L1 therapy. J Neuroinflammation. 2018;15(1):290.

13. Liang Z, Wong RP, Li LH, Jiang H, Xiao H, Li G. Development of pan-specific antibody against trimethyllysine for protein research. Proteome Sci. 2008;6:2.

14. Poulard C, Corbo L, Le Romancer M. Protein arginine methylation/demethylation and cancer. Oncotarget. 2016;7(41):67532-50.

15. Harrison MJ, Tang YH, Dowhan DH. Protein arginine methyltransferase 6 regulates multiple aspects of gene expression. Nucleic Acids Res. 2010;38(7):2201-16.

16. Hyllus D, Stein C, Schnabel K, Schiltz E, Imhof A, Dou Y, Hsieh J, Bauer UM. PRMT6-mediated methylation of R2 in histone H3 antagonizes H3 K4 trimethylation. Genes Dev. 2007;21(24):3369-80.

17. Teyssier C, Le Romancer M, Sentis S, Jalaguier S, Corbo L, Cavaillès V. Protein arginine methylation in estrogen signaling and estrogen-related cancers. Trends Endocrinol Metab. 2010;21(3):181-9.

18. Jiang N, Li QL, Pan W, Li J, Zhang MF, Cao T, Su SG, Shen H. PRMT6 promotes endometrial cancer via AKT/mTOR signaling and indicates poor prognosis. Int J Biochem Cell Biol. 2020;120:105681.

19. Avasarala S, Wu PY, Khan SQ, Yanlin S, Van Scoyk M, Bao J, Di Lorenzo A, David O, Bedford MT, Gupta V, et al. PRMT6 Promotes Lung Tumor Progression via the Alternate Activation of Tumor-Associated Macrophages. Mol Cancer Res. 2020;18(1):166-78.

20. Consortium GT. The Genotype-Tissue Expression (GTEx) project. Nat Genet. 2013;45(6):580-5.

21. Newman A, Liu C, Green M, Gentles A, Feng W, Xu Y, Hoang C, Diehn M, Alizadeh A. Robust enumeration of cell subsets from tissue expression profiles. Nature methods. 2015;12(5):453-7.

22. Ru B, Wong C, Tong Y, Zhong J, Zhong S, Wu W, Chu K, Wong C, Lau C, Chen I, et al. TISIDB: an integrated repository portal for tumor-immune system interactions. Bioinformatics. 2019;35(20):4200-2.

23. Bao Z, Chen H, Yang M, Zhang C, Yu K, Ye W, Hu B, Yan W, Zhang W, Akers J, et al. RNA-seq of 272 gliomas revealed a novel, recurrent PTPRZ1-MET fusion transcript in secondary glioblastomas. Genome research. 2014;24(11):1765-73.

24. Zhao Z, Meng F, Wang W, Wang Z, Zhang C, Jiang T. Comprehensive RNA-seq transcriptomic profiling in the malignant progression of gliomas. Scientific data. 2017;4:170024.

25. Subramanian A, Kuehn H, Gould J, Tamayo P, Mesirov J: GSEA-P: a desktop application for Gene Set Enrichment Analysis. Bioinformatics (Oxford, England) 2007, 23(23):3251-3253.

26. Lee Y, Ma H, Tan T, Ng S, Soong R, Mori S, Fu X, Zernicka-Goetz M, Wu Q. Protein arginine methyltransferase 6 regulates embryonic stem cell identity. Stem Cells Dev. 2012;21(14):2613-22.

27. Guccione E, Bassi C, Casadio F, Martinato F, Cesaroni M, Schuchlautz H, Lüscher B, Amati B. Methylation of histone H3R2 by PRMT6 and H3K4 by an MLL complex are mutually exclusive. Nature. 2007;449(7164):933-7. 
28. Hyllus D, Stein C, Schnabel K, Schiltz E, Imhof A, Dou Y, Hsieh J, Bauer U. PRMT6-mediated methylation of R2 in histone H3 antagonizes H3 K4 trimethylation. Genes Dev. 2007;21(24):3369-80.

29. Yu Z, Chen T, Hebert J, Li E, Richard S. Correction for Yu et al., "A Mouse PRMT1 Null Allele Defines an Essential Role for Arginine Methylation in Genome Maintenance and Cell Proliferation". Mol Cell Biol 2017, 37(17).

30. Luo Y, Xie C, Brocker CN, Fan J, Wu X, Feng L, Wang Q, Zhao J, Lu D, Tandon M, et al. Intestinal PPARalpha Protects Against Colon Carcinogenesis via Regulation of Methyltransferases DNMT1 and PRMT6. Gastroenterology. 2019;157(3):744-59 e744.

31. Wong TL, Ng KY, Tan KV, Chan LH, Zhou L, Che N, Hoo RLC, Lee TK, Richard S, Lo CM, et al. CRAF Methylation by PRMT6 Regulates Aerobic Glycolysis-Driven Hepatocarcinogenesis via ERK-Dependent PKM2 Nuclear Relocalization and Activation. Hepatology. 2020;71(4):1279-96.

32. Bindea G, Mlecnik B, Tosolini M, Kirilovsky A, Waldner M, Obenauf A, Angell H, Fredriksen T, Lafontaine L, Berger A, et al. Spatiotemporal dynamics of intratumoral immune cells reveal the immune landscape in human cancer. Immunity. 2013;39(4):782-95.

33. Schnell A, Schmidl C, Herr W, Siska P. The Peripheral and Intratumoral Immune Cell Landscape in Cancer Patients: A Proxy for Tumor Biology and a Tool for Outcome Prediction. Biomedicines 2018, 6(1).

34. Jarrold J, Davies C. PRMTs and Arginine Methylation: Cancer's Best-Kept Secret? Trends Mol Med. 2019;25(11):993-1009.

35. Li C, Liu T, Zhou B, Zhou Y, Yu H, Sun Y: Efficacy and safety analysis on dendritic cell-based vaccine-treated high-grade glioma patients: a systematic review and meta-analysis. OncoTargets and therapy 2018, 11:7277-7293.

36. Nagai Y, Ji M, Zhu F, Xiao Y, Tanaka Y, Kambayashi T, Fujimoto S, Goldberg M, Zhang H, Li B, et al. PRMT5 Associates With the FOXP3 Homomer and When Disabled Enhances Targeted p185 Tumor Immunotherapy. Frontiers in immunology. 2019;10:174.

37. Yang S, Cheng H, Huang Z, Wang X, Wan Y, Cai J, Wang Z. Circulating soluble neuropilin-1 in patients with early cervical cancer and cervical intraepithelial neoplasia can be used as a valuable diagnostic biomarker. Dis Markers. 2015;2015:506428.

38. Zeng, Lin H, Cui J, Liang W. TOX3 is a favorable prognostic indicator and potential immunomodulatory factor in lung adenocarcinoma. Oncol Lett. 2019;18(4):4144-52.

39. Su X, Zhang J, Fu C, Xiao M, Wang C. Recurrent Metastatic Penile Cancer Patient with Positive PD-L1 Expression Obtained Significant Benefit from Immunotherapy: A Case Report and Literature Review. OncoTargets therapy. 2020;13:3319-24.

40. Wang L, Ge J, Lan Y, Shi Y, Luo Y, Tan Y, Liang M, Deng S, Zhang X, Wang W, et al. Tumor mutational burden is associated with poor outcomes in diffuse glioma. BMC Cancer. 2020;20(1):213.

41. Juliano J, Gil O, Hawkins-Daarud A, Noticewala S, Rockne R, Gallaher J, Massey S, Sims P, Anderson A, Swanson K, et al: Comparative dynamics of microglial and glioma cell motility at the infiltrative margin of brain tumours. Journal of the Royal Society, Interface 2018, 15(139).

42. Luo M, Li Y, Guo H, Lin S, Chen J, Ma Q, Gu Y, Jiang Z, Gui Y. Protein Arginine Methyltransferase 6 Involved in Germ Cell Viability during Spermatogenesis and Down-Regulated by the Androgen Receptor. Int J Mol Sci. 2015;16(12):29467-81.

43. Nakakido M, Deng Z, Suzuki T, Dohmae N, Nakamura Y, Hamamoto R. PRMT6 increases cytoplasmic localization of p21CDKN1A in cancer cells through arginine methylation and makes more resistant to cytotoxic agents. Oncotarget. 2015;6(31):30957-67.

44. Koster R, di Pietro A, Timmer-Bosscha H, Gibcus J, van den Berg A, Suurmeijer A, Bischoff R, Gietema J, de Jong S. Cytoplasmic p21 expression levels determine cisplatin resistance in human testicular cancer. J Clin Investig. 2010;120(10):3594-605.

45. Sone K, Piao L, Nakakido M, Ueda K, Jenuwein T, Nakamura Y, Hamamoto R. Critical role of lysine 134 methylation on histone H2AX for $\mathrm{Y}-\mathrm{H} 2 \mathrm{AX}$ production and DNA repair. Nature communications. 2014;5:5691.

46. Hamamoto R, Saloura V, Nakamura Y. Critical roles of non-histone protein lysine methylation in human tumorigenesis. Nature reviews Cancer. 2015;15(2):110-24.

\section{Figures}


$\mathbf{a}$
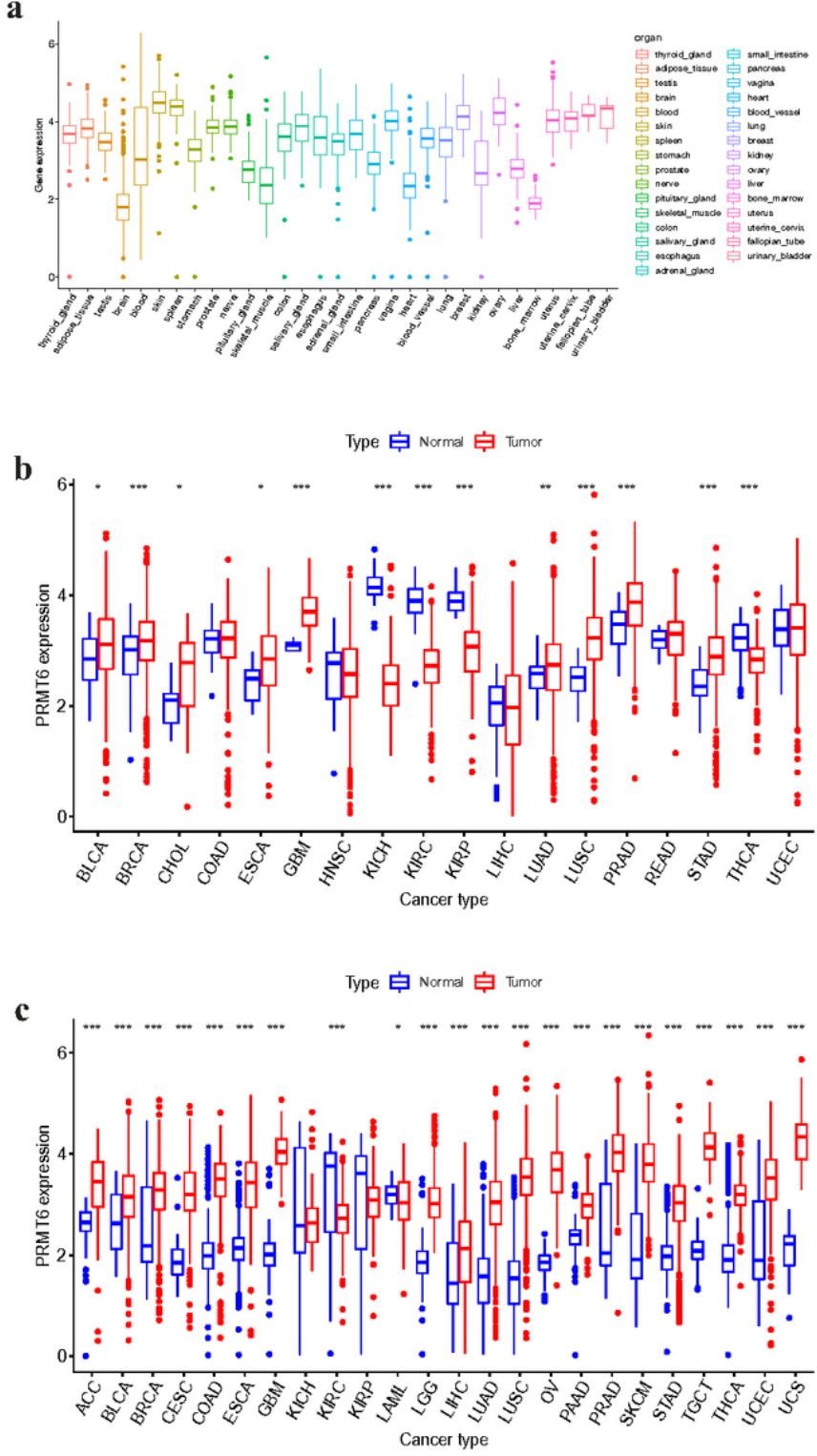

Figure 1

Expression and comparison of PRMT6 in different normal tissues and tumors. a. Expression of PRMT6 in 31 normal human tissues. b-c. Differences in expression of PRMT6 between normal tissues and paracancer. $(*: P<0.05$; $* \star: P<0.01$; $* \star \star: P<0.001)$. 


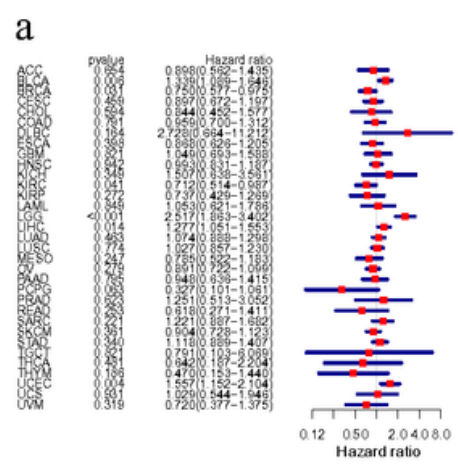

b

Cancer: LGG
PRMTE levels + high + low
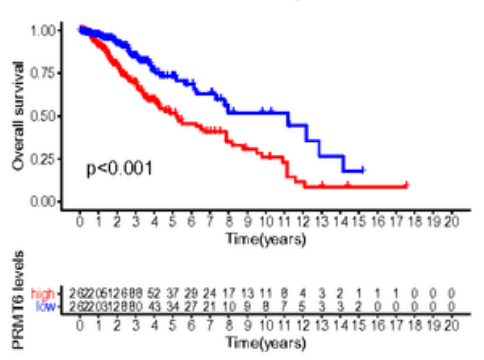

e

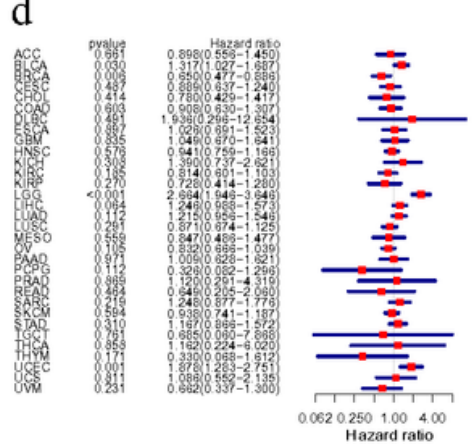

$\mathrm{h}$

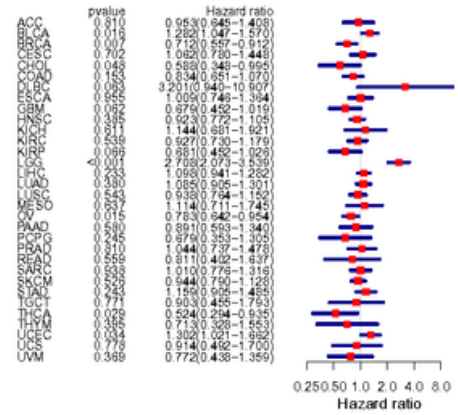

Cancer LGG

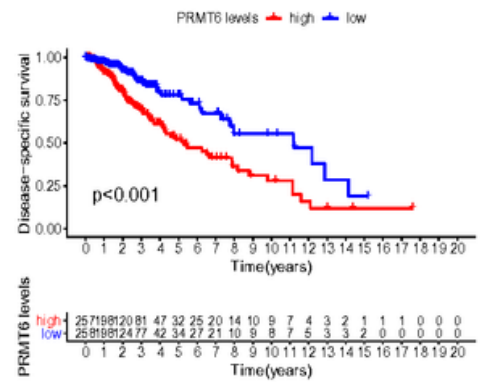

i

Cancer: LGG

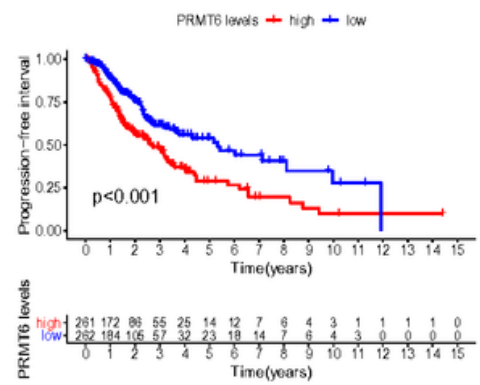

f

Cancer: LUAD

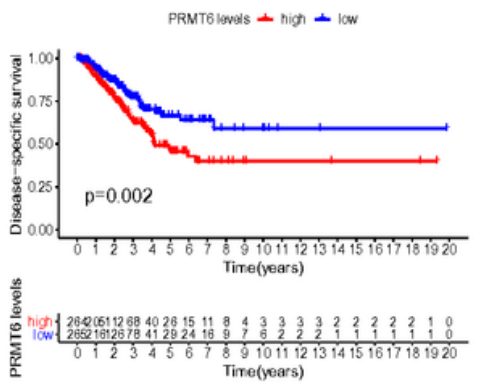

j

Cancer: LUAD

PRMT6 levels + nigh - low
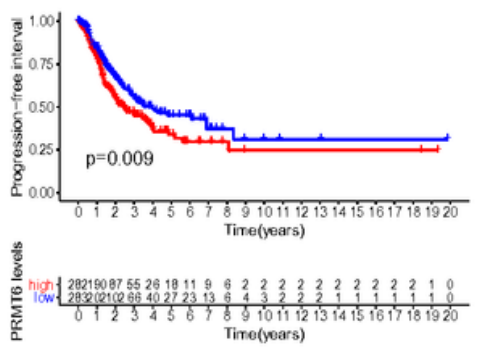

g

Cancer: UCEC

PRMTE lenels - high - low

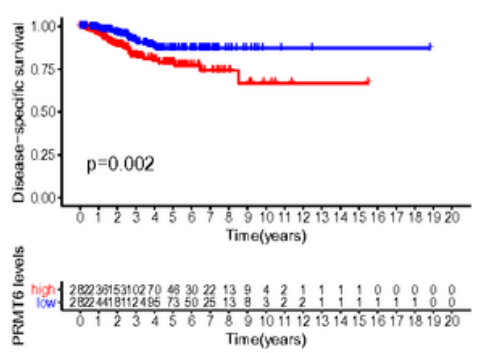

$\mathrm{k}$

Cancer: $C O A D$

PRMTElenels + high + low

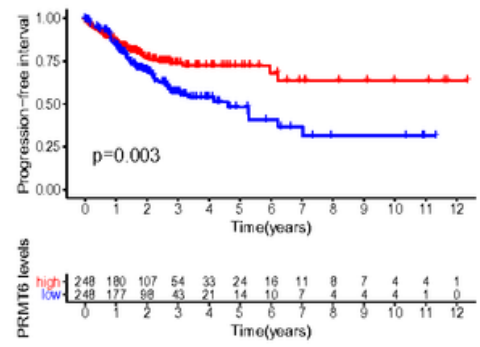

\section{Figure 2}

Analysis of PRMT6 expression and prognosis paracancer. (a.d.h). Forest map of the relationship between PRMT6 expression and prognosis in 33 tumors, OS,DSS and PFI, respectively. (b-c). In OS, the survival analysis curve consisted of high and low expression of PRMT6, and the tumors with statistical significance included LGG and UCEC. (e-g). In DSS, the survival analysis curve consisted of high and low expression of PRMT6, and the tumors with statistical significance included LGG, LUAD and UCEC. (i-k). In PFI, the survival analysis curve consisted of high and low expression of PRMT6, and the tumors with statistical significance included LGG, COAD and LUAD. 

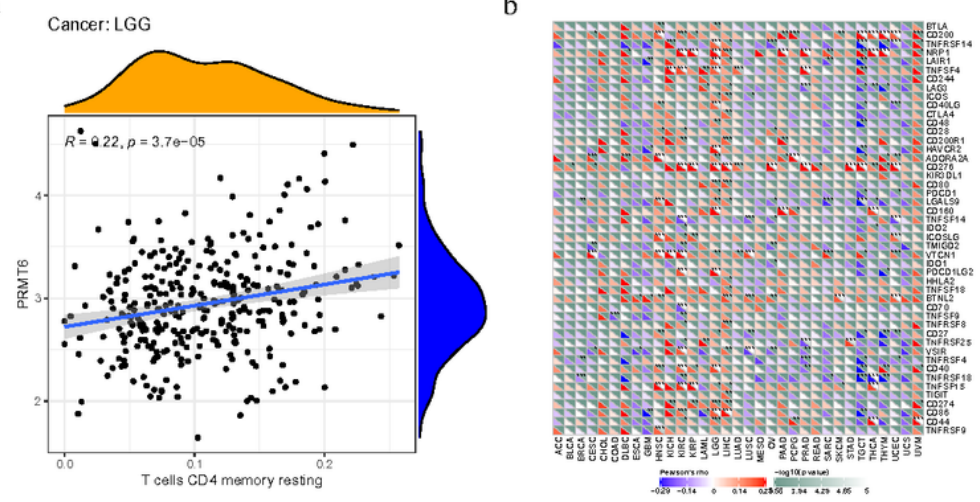

$\mathbf{c}$

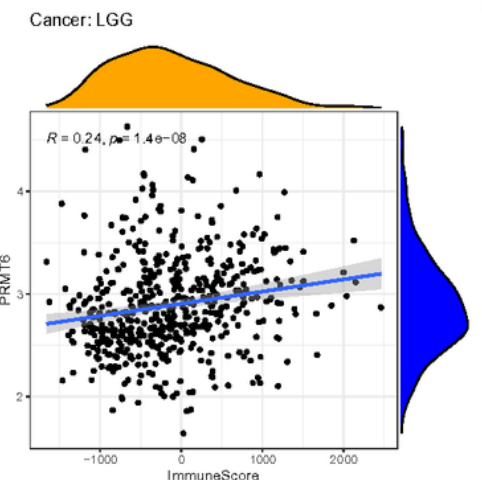

d Cancer: LGG
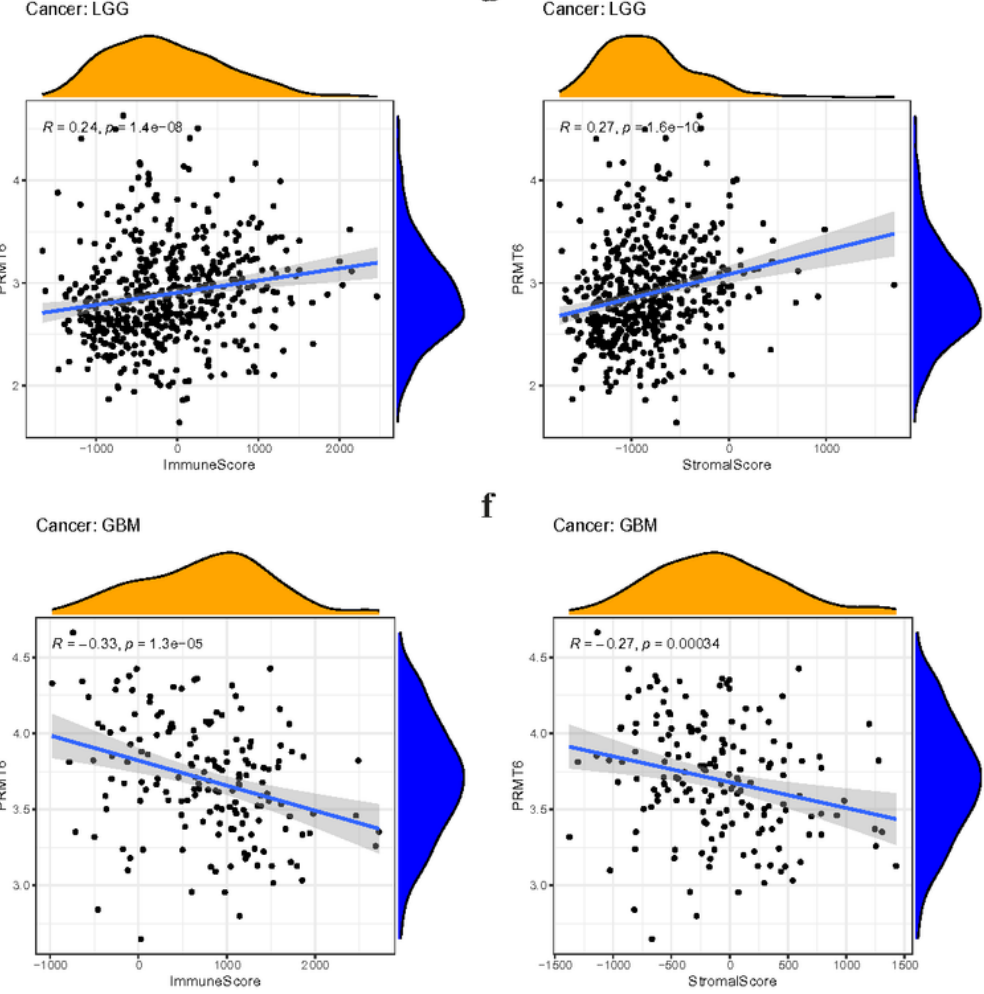

f

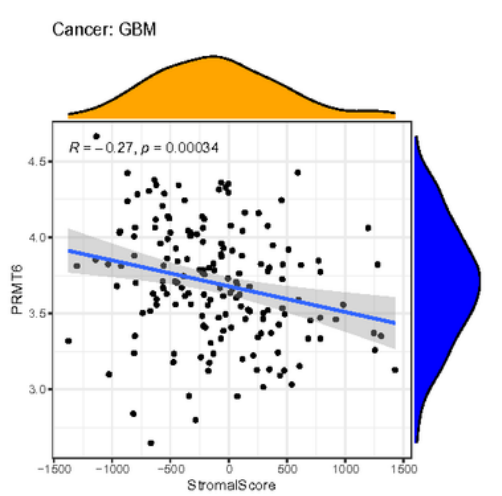

Figure 3

The relationship between PRMT6 expression and immune. a. Relationship between PRMT6 expression and immune cells in LGG. b. Correlation between PRMT6 expression and immune checkpoint. c-f. Correlations between PRMT6 expression and immunescore or stromascore in LGG and GBM. 
a

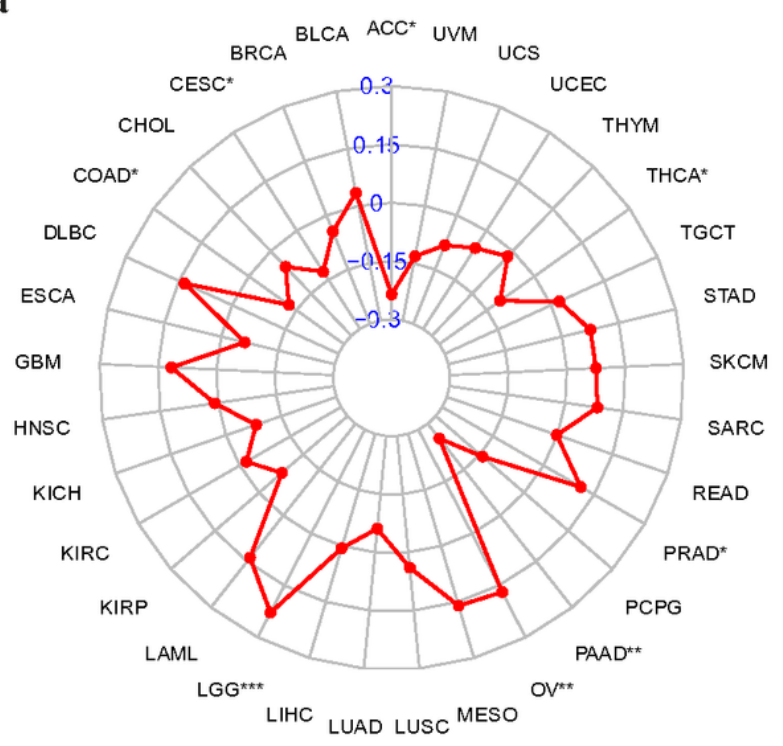

b

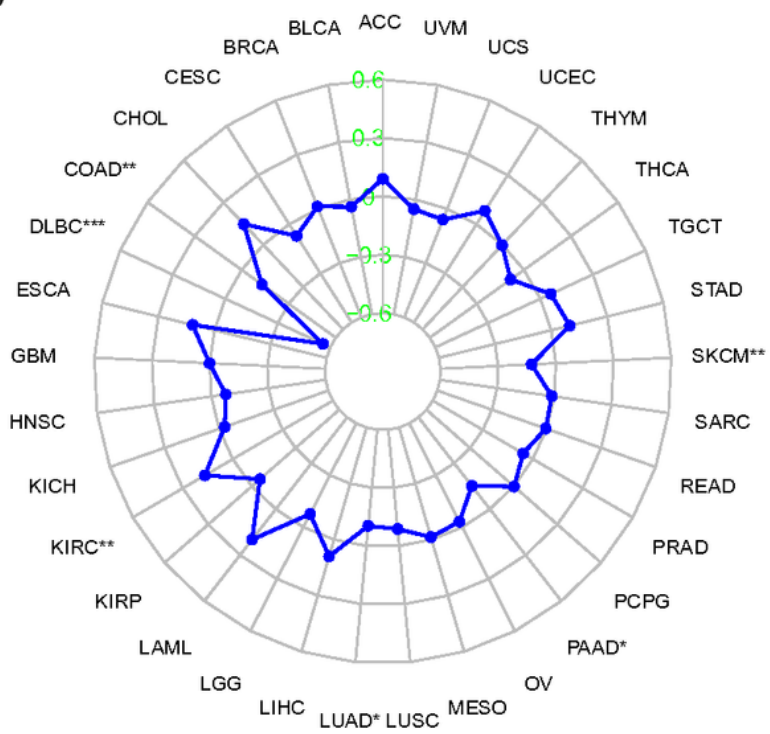

Figure 4

The relationship between PRMT6 expression and mutation. a. The expression of PRMT6 in 33 tumors was correlated with TMB. b. The expression of PRMT6 in 33 tumors was correlated with MSI. 

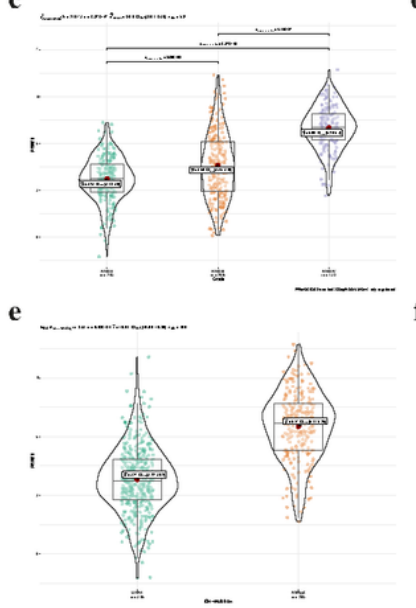

g

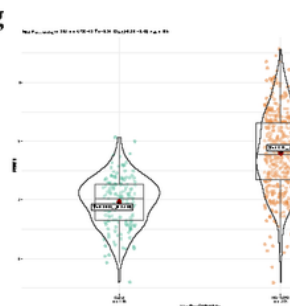

i

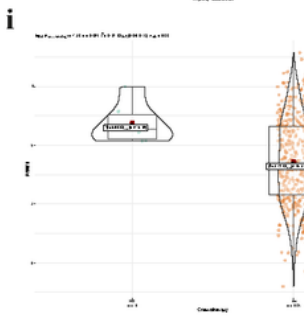

h b

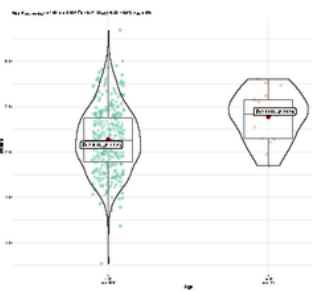

d

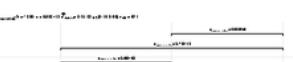

i
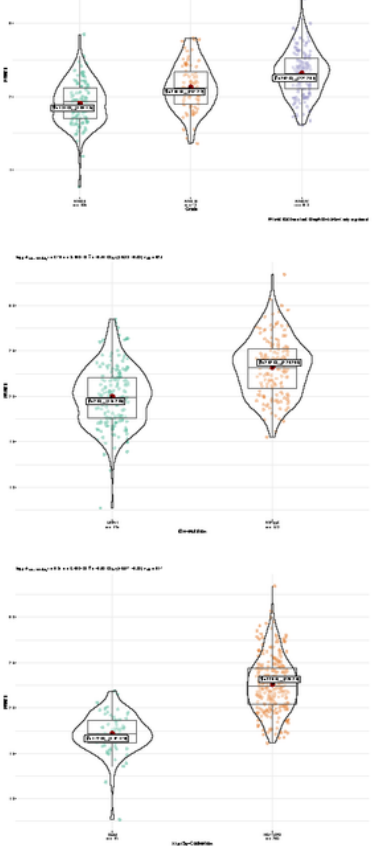

i.

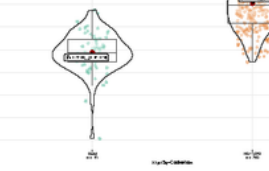

j

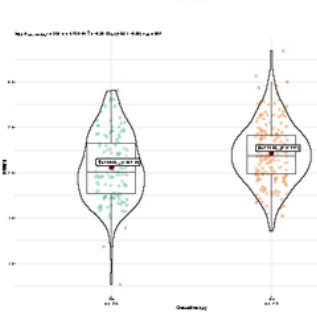

Figure 5

The relationship between PRMT6 expression and common clinical risk factors. a. c. e. g. i. The relationship between the two in the TCGA database. b. d. f. h. j. The relationship between the two in the CGGA database. 
a

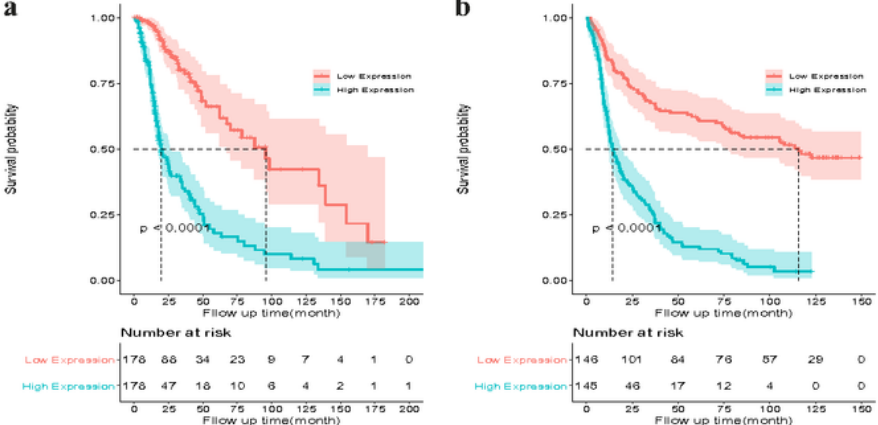

c

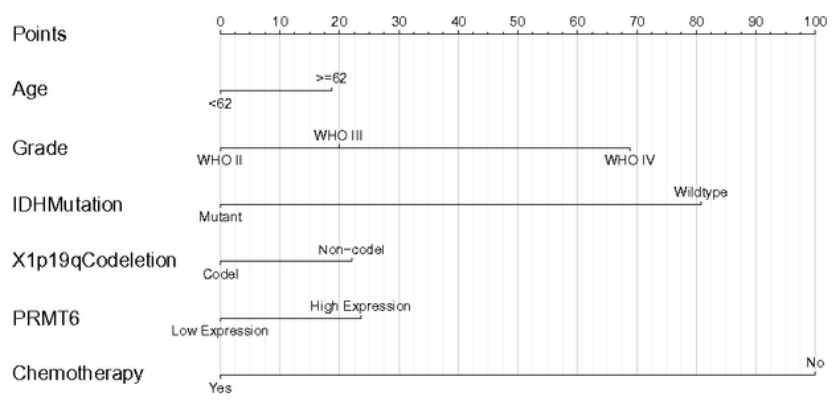

Total Points

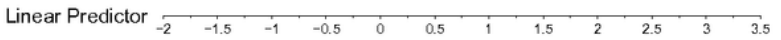

$\begin{array}{llllllllllll}\text { 1-year OS Probability } & 0.95 & 0.9 & 0.8 & 0.7 & 0.6 & 0.5 & 0.4 & 0.3 & 0.2 & 0.1\end{array}$

3-year OS Probability $\begin{array}{lllllllllll}0.9 & 0.8 & 0.7 & 0.6 & 0.5 & 0.4 & 0.3 & 0.2 & 0.1\end{array}$

d
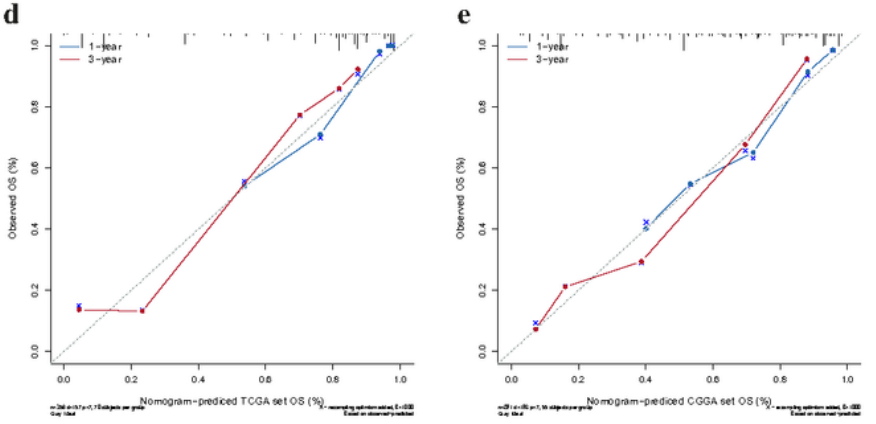

Figure 6

a. Survival analysis curve based on TCGA data. b. Survival analysis curve based on CGGA data. c. Nomogram based on TCGA data. d. Calibration curve of nomogram based on TCGA data. e. Calibration curve of nomogram based on CGGA data. 
a
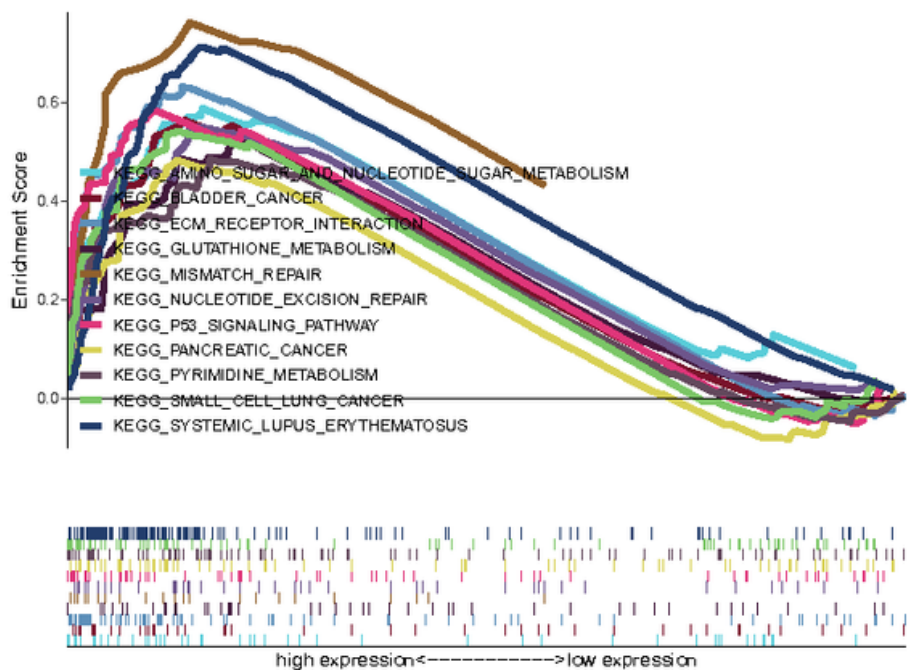

c

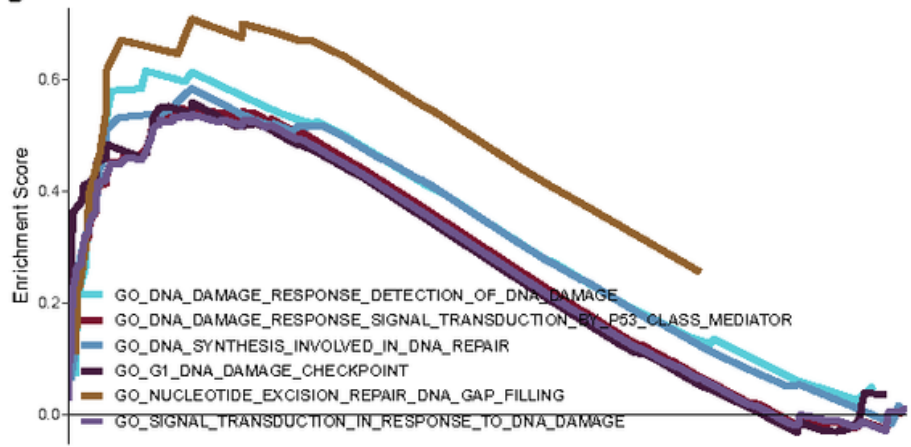

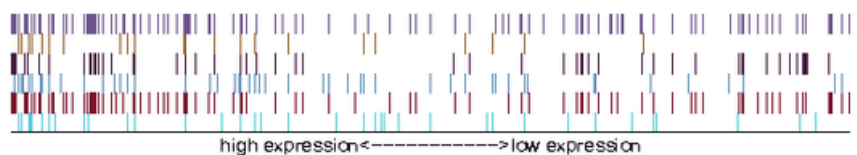

b
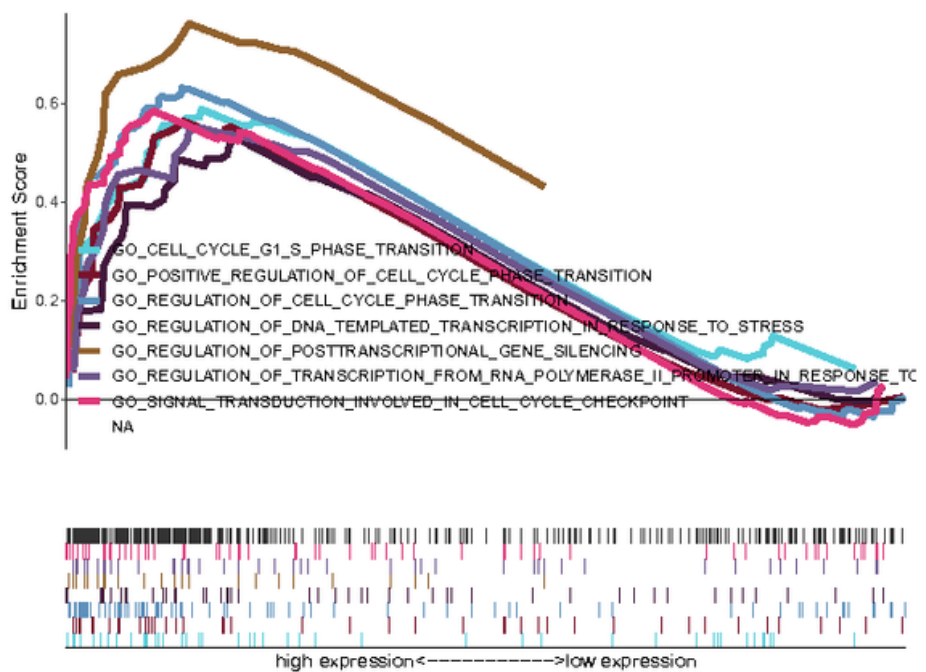

d

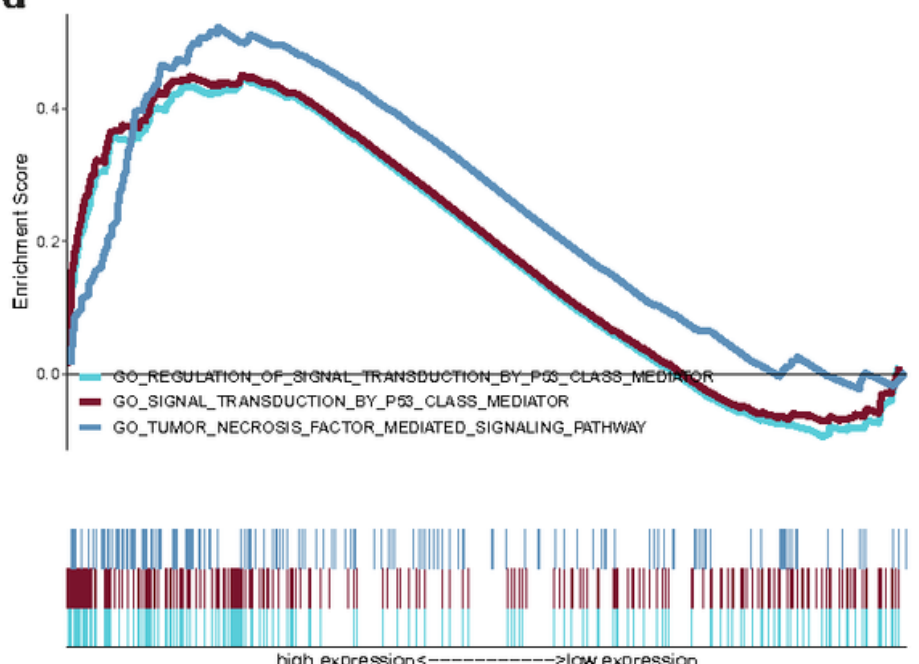

Figure 7

Results of gene enrichment analysis. a. Gene enrichment KEGG analysis. b. Gene enrichment GO analysis on the enrichment results of cell cycle regulation. c. Gene enrichment GO analysis on the enrichment results of DNA damage and repair. d. Gene enrichment GO analysis on the enrichment results of signal transduction. 

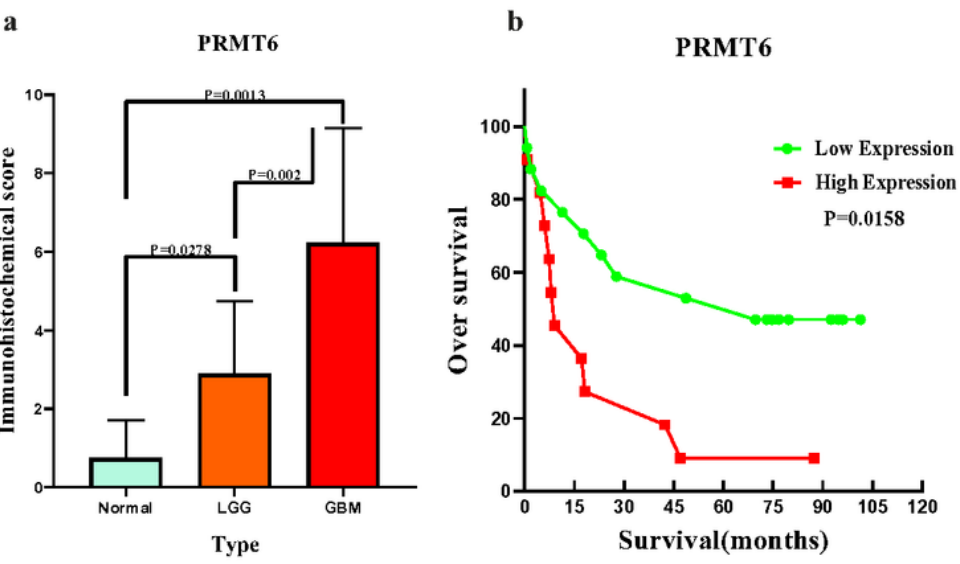

$\mathbf{c}$

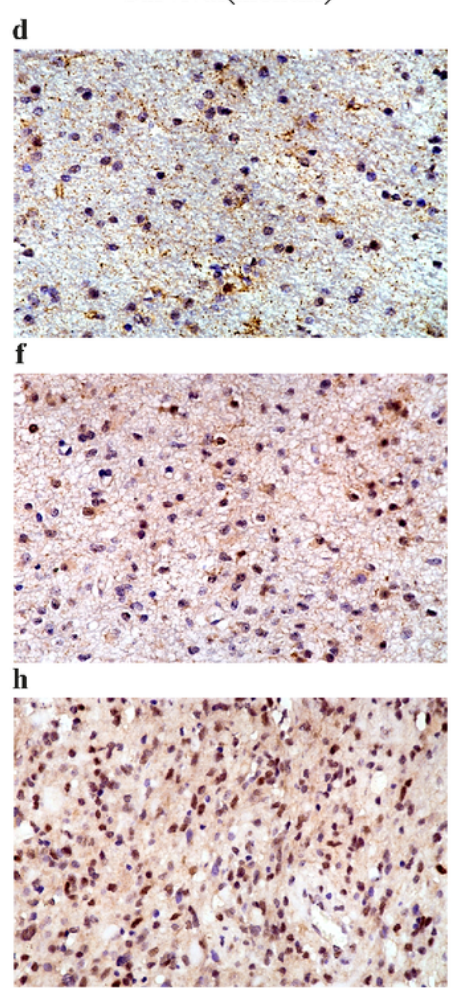

Figure 8

Immunohistochemical results. a. Histograms of PRMT6 scores and tissue types in immunohistochemical experiments. b. Survival analysis of high and low expression of PRMT6 in immunohistochemical experiments. c-d. Immunohistochemical staining sections of nomal paracancer tissue with PRMT6 were 200 - and 400 - fold microscopic images, respectively. e-f. Immunohistochemical staining sections of LGG with PRMT6 were 200 - and 400 - fold microscopic images, respectively. g-h. Immunohistochemical staining sections of GBM with PRMT6 were 200 - and 400 - fold microscopic images, respectively.

\section{Supplementary Files}

This is a list of supplementary files associated with this preprint. Click to download.

- Additionalfile1FigureS1.pdf

- Additionalfile2Figures2.pdf

- Additionalfile3FigureS3.pdf

- Additionalfile4FigureS4.pdf 